\title{
The case of equivalence of low and high energy constraints on Regge vector spectrum in AdS/QCD
}

\author{
S. S. Afonin and T. D. Solomko \\ Saint Petersburg State University, 7/9 Universitetskaya nab., St.Petersburg, \\ 199034, Russia
}

\begin{abstract}
The AdS/QCD models are believed to interpolate between low and high energy sectors of QCD. This belief is usually based on observations that many phenomenologically reasonable predictions follow from bounds imposed at high energies although the hypothetical range of applicability of semiclassical bottom-up holographic models is restricted by the gauge/gravity duality to low energies where QCD is strongly coupled. For testing the feasibility of high energy constraints it is interesting to calculate holographically some observable constants at low and high momenta independently and compare. We consider an AdS/QCD model describing the Regge-like linear spectrum of spin-1 mesons in a general form and show that under definite physical assumptions, the low-energy constraints on 2-point correlation functions lead to nearly the same numerical values for the parameters of linear radial spectrum as the high energy ones. The found approximate coincidence looks surprising in view of the fact that such a property for observables is natural for conformal theories while real strong interactions are not conformal.
\end{abstract}

\section{Introduction}

The bottom-up holographic models for QCD (often referred to as AdS/QCD models) have proven to be an interesting approach to the phenomenology of strong interactions [1]. Being inspired by the gauge/gravity duality in string theory [2,3], they boldly apply the holographic methods developed for conformal theories to the case of real QCD which is not conformal. A theoretical justification for such an extension is still lacking (see, however, the pioneering work [4]), nevertheless the bottom-up holographic approach resulted in construction of some useful phenomenological models which turned out to be 
unexpectedly successful [5-7] and triggered a large activity in the field (the Refs. [8 33] constitute only a tiny part).

According to the principles of gauge/gravity duality, the AdS/QCD models should be viewed as models for QCD constructed (i) in the large- $N_{c}$ limit [34,35] and (ii) in the low energy domain where QCD is strongly coupled. The point (i) formally implies that only 2-point correlation functions can be described self-consistently (the higher n-point functions vanish in the large- $N_{c}$ limit [35]) while the point (ii) ensures that a putative 5D dual gravitational theory is weakly coupled, hence, can be treated semiclassically. In phenomenological applications, however, one often weakens both requirements. For instance, the incorporation of Chern-Simons terms allows to describe various $\mathcal{O}\left(1 / N_{c}\right)$-effects of anomalies. Also the descriptions of spontaneous Chiral Symmetry Breaking (CSB) and hadron formfactors involve higher npoint correlators $[1,5,6]$. The applications to high energy QCD, which look speculative in view of (ii), turn out to be rather successful. As an example one can mention holographic derivations of QCD sum rules 21,23 25]. Among the practitioners of AdS/QCD models there is a widespread belief that these models efficiently interpolate between low energy and high energy QCD. In certain sense, they can be seen as a "meromorphization" of the perturbative QCD expression for 2-point correlation functions [1]. Since essentially the same is pursued in the large- $N_{c}$ QCD sum rules [36 47], the holographic analogues of these sum rules provide a very similar level of predictiveness.

The correct description of 2-point QCD correlators at large Euclidean momentum $Q$ (perturbative logarithm plus power corrections in $Q^{-2}$ ) is provided by the Soft-Wall (SW) holographic model [7] (an earlier variant was suggested in Ref. [20]). This model describes the Regge-like radial meson spectrum that in the case of spin-1 mesons reads $m_{n}^{2}=\mu^{2}(n+1)$, where $n=0,1,2, \ldots$ The SW model can be generalized towards inclusion of arbitrary intercept parameter $b, m_{n}^{2}=\mu^{2}(n+1+b)$ [25]. The necessity for non-zero intercept parameter $b$ is provided by the spectroscopy of light mesons. For example, consider the non-strange isosinglet states in the vector and axial-vector channels. According to the Particle Data [48], there are only three well-established $\omega$-mesons: $\omega(782), \omega(1420)$, and $\omega(1650)$. Taking their masses from [48] and ascribing them the "radial" quantum numbers $n=0,1,2$, we obtain the fit $\left(\right.$ in $\left.\mathrm{GeV}^{2}\right): m_{\omega}^{2}(n) \approx 1.1(n+0.7)$. In the axialvector sector, there is only one well-established state $f_{1}(1285)$ (another one, $f_{1}(1420)$, consists mostly of the strange quarks). As a motivated guess, let us use the non-confirmed states $f_{1}(1970)$ and $f_{1}(2230)$ [48]. We ascribe them the "radial" quantum numbers $n=2,3$ (the state corresponding to $n=1$ — the isoscalar partner of $a_{1}(1640)$ [48] — is not known). This gives the fit: $m_{f_{1}}^{2}(n) \approx 1.1(n+1.5)$. Thus we see that the correction to the vector 
intercept of standard SW model is at the level of $50 \%$. Note a remarkable fact that the slopes in the obtained linear fits are approximately equal.

The holographic sum rules yield definite predictions for the intercept $b$ in the vector and axial case. The aim of the present paper is to demonstrate how almost the same numerical predictions can be obtained in the opposite limit of low $Q^{2}$ expansion of correlators. This looks really surprising since the corresponding equations are very different from the case of high $Q^{2}$ expansion. In addition, we will reproduce an expression for the slope $\mu^{2}$ known from QCD sum rules.

As a byproduct of our analysis, we will show how the effects of CSB can be embedded into holographic models on the level of 2-point correlators, i.e. in agreement with (i). The usual bottom-up holographic description of CSB is based on merging of chiral effective field theory with AdS/QCD models [5, 6] that inevitably includes higher n-point functions. Such a phenomenological approach has been remarkably successful in the case of Hard Wall (HW) holographic model [5.6] (this model, however, does not reproduce the Reggelike spectrum and power-like corrections in high $Q^{2}$ expansion of correlators) but faced problems in simple realizations of SW ones. We will effectively take into account some CSB effect: 11 via different conditions on 2-point correlators at zero momentum using some physical motivations. Within this scheme, even the simplest SW holographic setup becomes a working model, at least after a certain reformulation.

The aforementioned reformulation constitutes another one, albeit secondary, objective of our work. We will argue that the replacement of enigmatic "dilaton background" by 5D mass depending on the fifth coordinate looks more natural from a physical viewpoint and leads to a simpler model. The proposals to introduce similar infrared modifications of 5D mass in AdS/QCD models have appeared in the literature from time to time since Ref. [26], we will motivate and exploit this formulation in a more systematic manner.

The present paper is aimed at analysis of conceptual points mentioned above which may help in further development of the theory of holographic QCD. We will not attempt to describe the meson spectrum "in the best possible way" - that problem is different in scope and often requires introduction of some non-linearities to Regge trajectories (see, e.g., the recent

\footnotetext{
${ }^{1}$ To be precise, we mean the mass splitting between the chiral partners, e.g., between the vector and axial-vector mesons, which is induced by the CSB, and the difference in the low-energy behavior of corresponding correlators. There is still not model-independent understanding of how these mass splittings are related with other features of CSB, such as the Gell-Mann-Oakes-Renner relation and low energy theorems. The incorporation of these features into our approach is a interesting task for the future.
} 
Refs. [50]; the non-linear radial meson spectra within the SW holographic approach were analyzed in Ref. [30|).

The paper is organized as follows. In order to make our analysis and arguments self-contained, in Section 2 we first briefly review the idea of AdS/QCD approach, reformulate the SW model and reproduce predictions for Regge spectral parameters of vector mesons from high $Q^{2}$ expansion of 2 -point correlator. The main results are contained in Section 3, where near the same predictions are obtained from the low $Q^{2}$ expansion. Some relevant discussions are given in Section 4 and we conclude in Section 5.

\section{Two-point vector correlators and OPE}

We first recall briefly the formalism of effective action and its holographic realization. Within the functional approach to quantum field theory, the primary object is the partition function $Z[\phi]$ of Green functions which has the physical meaning of vacuum-to-vacuum transition amplitude in the presence of external source $\phi, Z[\phi]=\left\langle 0_{\text {out }} \mid 0_{\text {in }}\right\rangle_{\phi}$. The effective action $S_{\text {eff }}\{\phi\}$ is defined by $Z[\phi]=\exp \left(i S_{\text {eff }}\{\phi\}\right)$. The coefficients of expansion of $S_{\text {eff }}\{\phi\}$ in the external field $\phi$ are the connected correlation functions of currents $J$ coupled to $\phi$. In the momentum space, the effective Lagrangian density of Lorentz invariant theory is

$$
\mathcal{L}_{\text {eff }}=\frac{1}{2} \hat{P} \operatorname{Tr}\left[\phi \Pi_{\phi}\left(q^{2}\right) \phi\right]+\mathcal{O}\left(\phi^{3}\right)
$$

where the term linear in $\phi$ disappears due to equation of motion, $\hat{P}$ denotes the corresponding polarization tensor if the field has Lorentz indices, and the scalar function $\Pi_{\phi}$ is called the two-point correlator of currents $J$ coupled to $\phi$ and is related to the full Green function of a field theory via

$$
\langle J J\rangle_{\phi}=\hat{P} \Pi_{\phi}
$$

In the large- $N_{c}$ limit of QCD, the correlator $\Pi_{\phi}$ is a meromorphic function and the higher n-point functions vanish [35]. It means in particular that in the limit $N_{c} \rightarrow \infty, \Pi_{\phi}$ has the structure of sum over infinite number of pole terms corresponding to contributions of infinitely narrow hadrons with quantum numbers of field $\phi$,

$$
\Pi_{\phi}\left(q^{2}\right) \sim \sum_{n=0}^{\infty} \frac{F_{n}^{2}}{q^{2}-m_{n}^{2}},
$$

where contact terms needed for regularization are omitted. 
In the bottom-up holographic approach, following the ideas of AdS/CFT correspondence one assumes the existence of 5D dual theory (in the sense of strong-weak duality) for QCD in the large- $N_{c}$ limit and tries to build a phenomenologically useful gravitational $5 \mathrm{D}$ model. This putative $5 \mathrm{D}$ theory is constructed in $5 \mathrm{D}$ Anti-de Sitter $\left(\mathrm{AdS}_{5}\right)$ space or asymptotically $\mathrm{AdS}_{5}$ space. The well-known reason for the choice of this space (at least when approaching boundary) is that only in this case the holographic principle for $4 \mathrm{D}$ field theories has chances to be naturally implemented - the 4D boundary of $\mathrm{AdS}_{5}$ is time-like (and only in the case of this space if we wish to have a homogeneous space of constant curvature) and has the appearance (at least after appropriate choice of coordinates) of 4D Minkowski flat space. Also the $\mathrm{AdS}_{5}$ space emerges naturally if one tries to "geometrize" the dilatation symmetry in a $4 \mathrm{D}$ conformal theory, i.e., to rewrite the scaling transformations of field theory operators as space-time transformations of some 5D fields [49]. For more specific motivations which are relevant to QCD see Ref. [4]. A convenient parametrization of the $\mathrm{AdS}_{5}$ metric is given by the Poincaré patch with the line element

$$
d s^{2}=\frac{R^{2}}{z^{2}}\left(\eta^{\mu \nu} d x_{\mu} d x_{\nu}-d z^{2}\right)
$$

where $R$ is the radius of $\mathrm{AdS}_{5}$ space and $z \geq 0$ represents the fifth holographic coordinate that has the physical meaning of inverse energy scale. The $4 \mathrm{D}$ Minkowski space becomes the ultraviolet boundary of $\mathrm{AdS}_{5}$ residing at $z=0$. The relation between a 4D gauge theory and its dual 5D gravitational theory schematically is given by a concise statement

$$
S_{\text {eff }}\{\phi(x)\}+J(x) \phi(x)=\left.S_{5 \mathrm{D}}^{\text {boundary }}(\phi(x, z))\right|_{\phi(x, 0) \doteq J(x)},
$$

where $\phi(x, 0)$ represents the UV boundary value of $\phi(x, z)$. If the $4 \mathrm{D}$ gauge theory is in the strong coupling regime, the $5 \mathrm{D}$ theory must be weakly coupled due to strong-weak duality. This general idea paved the way for building semiclassical 5D models which describe the low energy QCD and are often interpolated to higher energies.

We will analyze the case of transverse vector fields, the corresponding polarization tensor is

$$
\hat{P}=\eta_{\mu \nu}-\frac{q_{\mu} q_{\nu}}{q^{2}}
$$

The simplest action of SW holographic model for vector mesons can be written as $(M, N=0,1,2,3,4)$

$$
S_{5 \mathrm{D}}=\frac{1}{g_{5}^{2}} \int d^{4} x d z \sqrt{g} e^{\varphi}\left(-\frac{1}{4} F^{M N} F_{M N}+\frac{1}{2} m_{5}^{2} V^{N} V_{N}\right),
$$


where $F_{M N}=\partial_{M} V_{N}-\partial_{N} V_{M}$ and $\varphi$ is given below in (13). The usual conditions,

$$
\partial^{\mu} V_{\mu}=0, \quad V_{z}=0
$$

are imposed on physical $4 D$ excitations. The $5 \mathrm{D}$ coupling $g_{5}$ plays the role of normalization constant for the field $V_{M}$. The quadratic in field structure of action (7) ensures disappearance of three and higher point correlation functions as expected in the large- $N_{c}$ QCD.

In the bottom-up holographic constructions, the 5D fields are required to disappear at four-dimensional infinity $x_{\mu} \rightarrow 0$ but extend to "holographic infinity" $z \rightarrow 0$ (that corresponds to infinity along the holographic radial coordinate $r$ related to $z$ as $r=R^{2} / z$ ), i.e. the fields are to live not only in the $5 \mathrm{D}$ bulk but also on the UV boundary $z=\epsilon, \epsilon \rightarrow 0$. They are also required to vanish on the IR boundary $z=\infty$. With these requirements imposed, one can integrate (7) by parts and arrive at the action

$$
\begin{aligned}
S_{5 \mathrm{D}}=\frac{1}{2 g_{5}^{2}} \int d^{4} x d z V_{M}\left\{\partial_{P}\left[\sqrt{g} e^{\varphi}\left(g^{P S} g^{M N}-g^{P M} g^{S N}\right) \partial_{S}\right]\right. \\
\left.\quad+m_{5}^{2} \sqrt{g} e^{\varphi} g^{M N}\right\} V_{N}-\left.\frac{1}{2 g_{5}^{2}} \int d^{4} x \sqrt{g} e^{\varphi} g^{z z} g^{M N} V_{M} \partial_{z} V_{N}\right|_{z=\epsilon} .
\end{aligned}
$$

The dynamics in the dual 5D theory are governed by the classical Equation Of Motion (e.o.m.) that leaves only the surface term in the action (9),

$$
S_{\text {on-shell }}=-\left.\frac{1}{2 g_{5}^{2}} \int d^{4} x \sqrt{g} e^{\varphi} g^{z z} g^{M N} V_{M} \partial_{z} V_{N}\right|_{z=\epsilon} .
$$

The AdS/CFT prescription for 5D mass of $p$-form field reads 3

$$
m_{5}^{2} R^{2}=(\Delta-p)(\Delta+p-4),
$$

where $\Delta$ is the canonical dimension of operator dual to the field $V_{M}$ on the $\mathrm{AdS}_{5}$ boundary. For vector fields the prescription dictates

$$
m_{5}^{2} R^{2}=(\Delta-1)(\Delta-3)
$$

since the vector field is a $p=1$ form. The usual quark vector current $\bar{q} \gamma_{\mu} q$ has $\Delta=3$, hence, $m_{5}^{2}=0$. Vector operators of higher dimensions correspond to massive $5 \mathrm{D}$ vector fields.

The dilaton-like quantity $\varphi$ in the action (7) dictates a background. The standard SW holographic model is defined by 7

$$
\varphi=c z^{2}
$$


where the constant $c$ provides a mass scale and can be both positive and negative. The choice (13) yields the Regge-like spectrum [7],

$$
m_{n}^{2}=4|c|(n+1), \quad n=0,1,2, \ldots,
$$

and what is very important it leads to a correct analytical structure of OPE. The physical origin of (13) remains an open problem. As was first noticed in Ref. [23], the dilaton-like background can be absorbed into a infrared modification of $m_{5}^{2}$. Indeed, using the metric (4) and restriction (8) the non-vanishing term with $z$-derivative in action $(7)$ can be written as

$$
\sqrt{g} e^{c z^{2}} \partial^{z} V^{N} \partial_{z} V_{N}=\left(\frac{R}{z}\right)^{5} e^{c z^{2}} g^{z R} g^{N S} \partial_{R} V_{S} \partial_{z} V_{N}=\frac{R}{z} e^{c z^{2}}\left(\partial_{z} V_{N}\right)^{2}
$$

where the inverse to metric $g_{M N}$ in (4) tensor is $g^{M N}=\frac{z^{2}}{R^{2}} \eta^{M N}$ and we write only lower indices when contraction with flat metric is understood, e.g., $V_{N}^{2}=\eta^{M N} V_{M} V_{N}$. Now we redefine the field

$$
V_{N}=e^{-c z^{2} / 2} v_{N}
$$

and arrive at

$$
\frac{R}{z}\left(\partial_{z} v_{N}-c z v_{N}\right)^{2}=\sqrt{g}\left(\partial^{z} v^{N} \partial_{z} v_{N}+\frac{c^{2} z^{4}}{R^{2}} v^{N} v_{N}\right)-2 R c v_{N} \partial_{z} v_{N}
$$

We see that a $\mathcal{O}\left(z^{4}\right)$ mass term emerged. The last term will not contribute to the e.o.m.. It is easy to show, however, that for scalar and tensor cases the analogous term contains a $z$-dependent factor and does contribute to the e.o.m. resulting in $\mathcal{O}\left(z^{2}\right)$ mass term.

The standard SW model can be thus reformulated without $z$-dependent dilaton background (13) if we instead use the following ansatz for $z$-dependent $5 \mathrm{D}$ mass,

$$
m_{5}^{2}(z) R^{2}=a+b z^{2}+c^{2} z^{4},
$$

which stays in the action

$$
S_{5 \mathrm{D}}=\frac{1}{2 g_{5}^{2}} \int d^{4} x d z \sqrt{g}\left(-\partial^{M} v^{N} \partial_{M} v_{N}+m_{5}^{2}(z) v^{N} v_{N}\right),
$$

Any infrared modifications of dilaton background or metric can be translated into corresponding modifications of (18). The constant $a$ must be identified with the AdS/CFT prescription (12), the further terms are infrared corrections. It should be emphasized that the given corrections do not violate this prescription because it is formulated on the UV boundary, $z \rightarrow 0$, only 
(the Ref. [49] contains a nice discussion of this point). For this reason any correction to (12) disappearing in the limit $z \rightarrow 0$ is compatible with the AdS/CFT prescription. On the other hand, arbitrary modifications of $z$ dependence in (18) will, generally speaking, spoil the structure of standard OPE for two-point correlators. This issue is closely related with the known fact that non-linear corrections to Regge-like spectrum (14) after summation over resonances in (3) generically lead to analytical structures incompatible with the OPE 45]. The compatibility can be achieved only if such corrections decrease with $n$ exponentially or faster [45,46]. A form of potential in the corresponding e.o.m. (written in a Schrödinger-like form) that would generate these corrections is unknown. In the present study, we will adhere to the ansatz (18) describing the linear spectrum in the most general form. Aside from reproducing correctly the analytical structure of OPE, the case of linear spectrum has the advantage of being exactly solvable.

For phenomenological description of real meson spectra with different quantum numbers one should introduce different intercepts in the spectrum (14). Within the SW model, this can be achieved by different infrared modifications of dilaton background or AdS metric. This fine tuning looks as if we constructed different dual models for different quantum numbers. The reformulation above looks nicer in this respect: A dual holographic model is unique but infrared modifications of $m_{5}^{2}$ are different. Note that the parameter $c$ in (18) is independent of quantum numbers since it dictates the slope of radial trajectories which phenomenologically is indeed approximately universal [51 56]. The given universality seems to be an important consequence of confinement and appears naturally in hadron string approaches and some related quark models [57 66]. The intercept will be determined by parameters $a$ and $b$. The first one can be fixed by (12). One of our goals will be determination of the second intercept parameter $b$.

The e.o.m. ensuing from the action $(19)$ with $m_{5}^{2}(z)$ from (18) after $4 \mathrm{D}$ Fourier transform

$$
v_{\mu}(q, z)=\int d^{4} x e^{i q x} v_{\mu}(x, z),
$$

takes the form

$$
\left[-q^{2}-z \partial_{z}\left(\frac{1}{z} \partial_{z}\right)+\frac{m_{5}^{2}(z) R^{2}}{z^{2}}\right] v_{\mu}(q, z)=0 .
$$

In the present work, we will consider the case $a=0$ in (18). This is an important case of twist 2 vector current according to relation $(12)$ and is the most studied in the literature ${ }^{2}$. In order to make comparisons with the

\footnotetext{
${ }^{2}$ The twist 2 operators, $\tau=\Delta-J=2$, correspond to operators of lowest scaling
} 
OPE in QCD, we must calculate the two-point correlator in Euclidean space, i.e. introducing Euclidean momentum $Q^{2}=-q^{2}$. Following the standard holographic procedure, we should find the solution of Eq. (21) in the form

$$
v_{\mu}(q, z)=v_{\mu}(q) v(q, z),
$$

with the boundary condition $v(q, 0)=1$. Then $v_{\mu}(q)$ can be interpreted as the source. The corresponding solution for the scalar shape function $v(q, z)$, satisfying also $v(q, \infty)=0$, is (we pass to Euclidean space in what follows)

$$
v(\mathcal{Q}, \zeta)=\Gamma\left(1+\mathcal{Q}^{2}+\beta\right) e^{-\zeta^{2} / 2} U\left(\mathcal{Q}^{2}+\beta, 0 ; \zeta^{2}\right),
$$

where $U$ is the Tricomi confluent hypergeometric function and for simplicity of further relations we defined the dimensionless quantities

$$
\mathcal{Q}^{2}=\frac{Q^{2}}{4|c|}, \quad \beta=\frac{b}{4|c|}, \quad \zeta^{2}=|c| z^{2} .
$$

Note that the Eq. 21) has the second solution which, however, diverges as $\zeta \rightarrow \infty$

$$
v_{2}(\mathcal{Q}, \zeta) \sim \zeta^{2} e^{-\zeta^{2} / 2} L_{-\left(1+\mathcal{Q}^{2}+\beta\right)}^{1}\left(\zeta^{2}\right) \sim e^{\zeta^{2} / 2} \zeta^{2\left(\mathcal{Q}^{2}+\beta\right)} .
$$

Here $L_{S}^{1}(x)=(1+S){ }_{1} F_{1}(-S, 2, x)$ denotes the corresponding Laguerre function. We discard this solution since it does not satisfy the holographic "regularity in the bulk" condition [3]. The solution (23) is regular in the bulk as $U\left(\mathcal{Q}^{2}+\beta, 0 ; \zeta^{2}\right) \sim \zeta^{-2\left(\mathcal{Q}^{2}+\beta\right)}$ at large $\zeta$.

Inserting (22) into the action 100 and taking the second functional derivative with respect to $v_{\mu}(q)$ we get the usual AdS/QCD expression for the vector 2-point correlator in Euclidean space,

$$
\Pi_{V}\left(\mathcal{Q}^{2}\right)=\lim _{\zeta \rightarrow 0}\left(-\frac{R}{g_{5}^{2}} \frac{\partial_{\zeta} v(\mathcal{Q}, \zeta)}{\zeta}\right)
$$

The expansion of solution $(23)$ at $\zeta \rightarrow 0$ yields

$$
v(\mathcal{Q}, \zeta)=1+\left\{\left(\mathcal{Q}^{2}+\beta\right)\left[\ln \zeta^{2}+\psi\left(1+\mathcal{Q}^{2}+\beta\right)+2 \gamma-1\right]-\frac{1}{2}\right\} \zeta^{2},
$$

dimension at a fixed spin $J$. Usually they play the decisive role in QCD dynamics. For instance, they give the dominant contribution in the analysis of hadronic deep inelastic scattering via the OPE 11. Also they interpolate the hadron states in the QCD sum rules $[68$ and in the lattice simulations. In the conformal field theory, they correspond to primary operators, i.e., realize the irreducible representations of conformal group and, thus, according to the AdS/CFT correspondence, are dual to 5D fields falling into irreducible representations of $\mathrm{AdS}_{5}$ space-time symmetry. 
where $\psi$ denotes the digamma function and $\gamma \approx 0.577$ is the Euler's constant.

Following the standard procedure, we should substitute (27) into (26) and take the limit $\zeta \rightarrow 0$. The logarithmic term will give the divergent contribution proportional to $\left(\mathcal{Q}^{2}+\beta\right) \ln \zeta^{2}$ which is interpreted as a contact term and discarded. This regularization is defined up to adding an arbitrary constant (or polynomial of $\mathcal{Q}^{2}$ ) which fixes the renormalization scheme. The high-energy predictions are scheme-independent. We will be interested, however, in low-energy predictions. In the latter case, adding arbitrary constant can change predictions in an arbitrary manner. We should thus fix a specific renormalization scheme that is the most adequate to the physical problem under consideration. Below we motivate such a scheme.

First of all, let us proceed more accurately following the relevant prescriptions of Holographic Renormalization (HR) [67]. The first step is to define the subtracted action as the sum of regularized and counterterm actions,

$$
S_{\text {sub }}[v(q, \epsilon) ; \epsilon]=S_{\text {reg }}[v(q, 0) ; \epsilon]+S_{\text {ct. }}[v(q, \epsilon) ; \epsilon] .
$$

The counterterm action $S_{\text {ct. }}$ must absorb the arising infinities such that the renormalized on-shell action $S_{\text {ren }}$ defined as

$$
S_{\text {ren }}[v(q, 0)]=\lim _{\epsilon \rightarrow 0} S_{\text {sub }}[v(q, \epsilon) ; \epsilon],
$$

is finite. In order to obtain correlation functions one varies $S_{\text {sub }}$, taking $\epsilon \rightarrow 0$ only at the end of the calculation. For this reason the distinction between $S_{\text {sub }}$ and $S_{\text {ren }}$ is important.

With our definitions and notations, the action (10) takes the form

$$
S=-\frac{R}{2 g_{5}^{2}} \int d^{4} x v_{\mu}(\mathcal{Q}) v_{\nu}(\mathcal{Q}) \eta^{\mu \nu}\left(\frac{1}{\zeta} v(\mathcal{Q}, \zeta) \partial_{\zeta} v(\mathcal{Q}, \zeta)\right)_{\zeta=\epsilon}
$$

For finding the structure of $S_{\text {ct. }}$ we should rewrite the near-boundary expansion (27) as

$$
v(\mathcal{Q}, \zeta)=v_{(0)}+\zeta^{2}\left(v_{(2)}+\tilde{v}_{(2)} \ln \zeta^{2}\right)+\mathcal{O}\left(\zeta^{4}\right) .
$$

According to the HR formalism [67], the coefficients $v_{(i)}$ (which in coordinate space represent certain functions of $x, v_{(i)}=v_{(i)}(x)$ ) have the following interpretation. $v_{(0)}$ is the field theory source. $\tilde{v}_{(2)}$ is related to conformal anomaly; it is a local function of $v_{(0)}$ and can be determined by the nearboundary analysis of e.o.m. The coefficient $v_{(2)}$ is a non-local function of $v_{(0)}$ in the coordinate space (they cannot be related by taking a finite number of derivatives), it is undetermined by the near-boundary analysis of e.o.m. and can be obtained only from the full solution in the bulk. 
The next step is to substitute (31) back to (30), the key factor will be

$$
\left(\frac{1}{\zeta} v \partial_{\zeta} v\right)_{\zeta=\epsilon}=2 v_{(0)}\left(v_{(2)}+\tilde{v}_{(2)}+\tilde{v}_{(2)} \ln \epsilon^{2}\right)+\ldots
$$

This expression dictates the structure of the counterterm action - it must cancel the logarithmic divergence near the UV boundary. Thus we obtain

$$
S_{\text {ct. }}=\frac{R}{g_{5}^{2}} \int d^{4} x v_{\mu}(\mathcal{Q}) v_{\nu}(\mathcal{Q}) \eta^{\mu \nu} v_{(0)} \tilde{v}_{(2)} \ln \epsilon^{2}+\text { Const }
$$

where a choice of Const fixes the renormalization scheme. Strictly speaking, one should invert the relations $v_{(0)}(v)$ and $\tilde{v}_{(2)}(v)$ and write $S_{\text {ct. }}$ in terms of $v$ because in the coordinate space it is $v(x, \epsilon)$ that defines the field $v_{\mu}$ (see (20) and $(22)$ ) transforming as a vector under bulk diffeomorphisms at $\zeta=\epsilon$. But this detail will not be essential for us.

In the minimal subtraction scheme, the subtracted action becomes thus proportional to $v_{(0)}\left(v_{(2)}+\tilde{v}_{(2)}\right)$ according to $(32)$. There is still the freedom to add a finite counterterm. This corresponds to scheme dependence in the field theory. It may happen that a physical problem under consideration dictates unambiguously which finite counterterm should be added [67]. We are of the opinion that this is relevant for our model as well. Let us recall a physical interpretation that is often ascribed to the subtraction of UV infinities in the field theory: One subtracts the contribution of a region lying outside the region of validity of the field theory, i.e. where the field theory needs a UV completion. A similar philosophy can be applied to the bottomup holographic model - in the ultraviolet limit $z \rightarrow 0$, the applicability of semiclassical holographic models to QCD looks questionable and the whole approach seems to need modifications (see, e.g., discussions in Refs. 27 29]). Keeping this in mind, we observe that the contribution from finite term $\tilde{v}_{(2)}$ also emerges from that UV region, hence, it is reasonable to subtract the given finite term as well ${ }^{3}$. This fixes our subtraction scheme: We retain only term $v_{(2)}$ that arises from dynamics in the bulk and subtract all terms stemming from the UV region where our model is viewed us inapplicable. All constants appearing in $v_{(2)}$ are considered as prediction of the model we cannot modify them manually since this would cause an arbitrary change of low-energy predictions.

On the operational level, the UV diverging contribution proportional to $\zeta^{2} \ln \zeta^{2}$ appears because the Tricomi function $U\left(S, 0 ; z^{2}\right)$ is not holomorphic

\footnotetext{
${ }^{3}$ We would remark also that subtraction of both terms proportional to $\tilde{v}_{(2)}$ removes a potential double counting in the following sense: These terms are related with conformal anomalies [67, i.e. with generation of a scale at a quantum level, but a scale is already introduced by hand in the model.
} 
in the point $z=0$. Our regularization is equivalent to subtraction of this singularity, the regularized solution becomes holomorphic at $z=0$.

Inserting the regularized expansion 27 into $(26)$ we obtain the two-point vector correlator in our model,

$$
\Pi\left(\mathcal{Q}^{2}\right)=-\frac{2 R}{g_{5}^{2}}|c|\left\{\left(\mathcal{Q}^{2}+\beta\right)\left[\psi\left(1+\mathcal{Q}^{2}+\beta\right)+2 \gamma-1\right]-\frac{1}{2}\right\} .
$$

This correlator is plotted in Fig. 1 for some typical values of parameters.

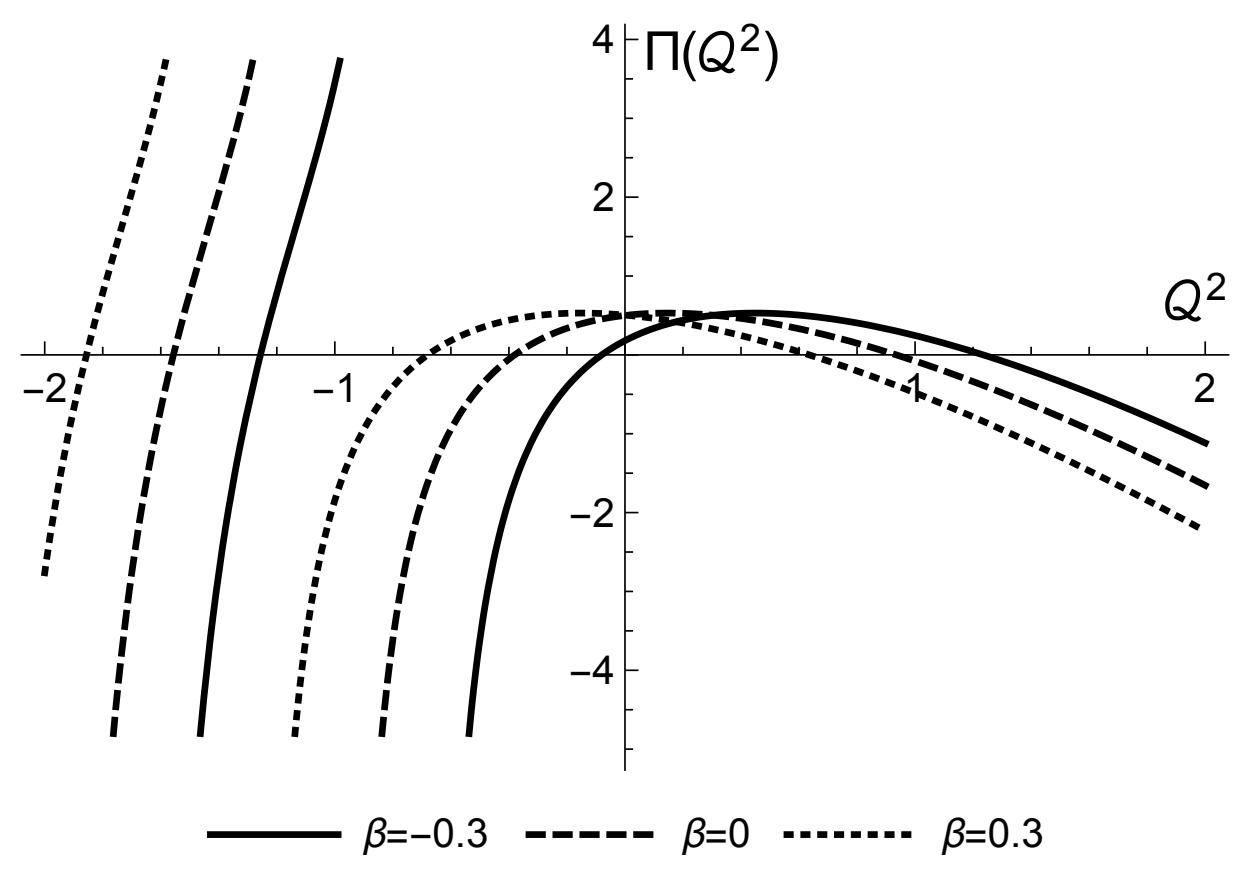

Figure 1: The two-point vector correlator (34) for three typical values of intercept parameter $\beta$ (see the discussions after Eq. 42 ). The general factor $\frac{2 R|c|}{g_{5}^{2}}$ is set to unity. In the time-like region of dimensionless momentum squared $\mathcal{Q}^{2}$, the plot extends to the position of the first pole (see (35)).

The expression (34) at $\beta=0$ coincides with the result of "No-wall" holographic model of Ref. [23]. In fact this expression can be obtained directly from the $\beta=0$ case by observing that $b \neq 0$ corresponds just to the shift of momentum squared $q^{2} \rightarrow q^{2}-b$ in the e.o.m. (21), i.e. $\mathcal{Q}^{2} \rightarrow \mathcal{Q}^{2}+\beta$.

The digamma function in (34) has poles at

$$
-\mathcal{Q}_{n}^{2}=\frac{m_{n}^{2}}{4|c|}=n+1+\beta, \quad n=0,1,2, \ldots,
$$


which correspond to the mass spectrum of the model. The substitution of known pole representation for this function,

$$
\psi(1+x)=\sum_{n=1}^{\infty} \frac{1}{n}-\sum_{n=1}^{\infty} \frac{1}{n+x}-\gamma
$$

into (34) leads to the expected analytical structure (3) written in the Euclidean space.

With the help of asymptotic representation of digamma function for large argument $x \rightarrow \infty$,

$$
\psi(1+x) \simeq \ln x+\frac{1}{2 x}-\frac{1}{12 x^{2}}+\mathcal{O}\left(x^{-3}\right),
$$

we can expand (34) at large $\mathcal{Q}^{2}$,

$$
\Pi\left(\mathcal{Q}^{2}\right) \simeq-\frac{2 R}{g_{5}^{2}}|c|\left\{\left(\mathcal{Q}^{2}+\beta\right) \ln \mathcal{Q}^{2}+\frac{\beta^{2}-1 / 6}{2 \mathcal{Q}^{2}}+\frac{\beta\left(1-2 \beta^{2}\right)}{12 \mathcal{Q}^{4}}+\ldots\right\},
$$

where the contact and $\mathcal{O}\left(\mathcal{Q}^{-6}\right)$ terms are omitted. The expansion $(38)$ only partly coincides with the result of generalized SW model of Ref. [25], where the arbitrary intercept $\beta$ in the linear spectrum (35) was introduced via the modified dilaton background $e^{c z^{2}} \rightarrow U^{2}\left(\beta, 0 ; c z^{2}\right) e^{c z^{2}}$.

The relevant form of $\mathrm{OPE}$ in $\mathrm{QCD}$ in the chiral and large- $N_{c}$ limits reads 68

$$
\Pi^{O P E}\left(\mathcal{Q}^{2}\right) \simeq-\frac{N_{c}}{6 \pi^{2}}|c| \mathcal{Q}^{2} \ln \mathcal{Q}^{2}+\frac{\left\langle\frac{\alpha_{s}}{\pi} G^{2}\right\rangle}{96|c| \mathcal{Q}^{2}}+\frac{\xi}{9} \frac{\pi \alpha_{s}\langle\bar{q} q\rangle^{2}}{8 c^{2} \mathcal{Q}^{4}}+\ldots,
$$

where $\left\langle\frac{\alpha_{s}}{\pi} G^{2}\right\rangle$ and $\langle\bar{q} q\rangle$ are the gluon and quark condensates, respectively, and $\xi$ depends on the space and charge parities $(\xi=-7$ for the vector case and $\xi=11$ for the axial-vector one). The omitted constant contribution in (39) (part of contact terms) allows to change the renormalization scale $\mu$ in the perturbative logarithm, we used this freedom to set $\mu^{2}=4|c|$.

The expansions (38) and (39) can be now matched. The matching of coefficients in front of the leading logarithms gives the standard normalization factor for the $5 \mathrm{D}$ vector fields,

$$
\frac{R}{g_{5}^{2}}=\frac{N_{c}}{12 \pi^{2}}
$$

The matching of $\mathcal{O}\left(\mathcal{Q}^{-2}\right)$ terms relates the gluon condensate to the spectral parameters as

$$
\left\langle\frac{\alpha_{s}}{\pi} G^{2}\right\rangle=\frac{N_{c}}{2 \pi^{2}}\left(\frac{1}{6}-\beta^{2}\right)(4 c)^{2} .
$$


As was shown in Ref. [25], a good agreement for the phenomenological slope of radial trajectories 51,56

$$
4|c| \approx 1.2 \mathrm{GeV}^{2}
$$

is achieved for the intercept parameter $|\beta| \approx 0.3$, where $\beta=\mp 0.3$ refers to the vector and axial cases, correspondingly. The arguments were the following: (i) $|\beta| \approx 0.3$ leads to a correct value of the gluon condensate in relation (41), $\left\langle\frac{\alpha_{s}}{\pi} G^{2}\right\rangle \approx(360 \mathrm{MeV})^{4}$; (ii) $\beta \approx-0.3$ gives a satisfactory description the spectrum of radially excited $\omega$-mesons within the accuracy of large- $N_{c}$ limit; (iii) $\beta \approx-0.3$ reproduces the observed electromagnetic decay width of $\omega$ meson; (iv) $\beta \approx 0.3$ describes reasonably the spectrum of radially excited axial $f_{1}$-mesons. The description of spectrum of excited isovector $\rho$ and $a_{1}$ mesons with $|\beta| \approx 0.3$ is even better. In addition, a remarkable qualitative agreement takes place: The first non-perturbative correction to the parton logarithm does not depend on parity while the next one is of opposite sign for different parities.

The quark effects are not incorporated into the holographic model under consideration. For this reason it is not surprising that the quark contributions like $\mathcal{O}\left(\mathcal{Q}^{-4}\right)$ and $\beta \ln \mathcal{Q}^{2}$ do not match. The former can have a sizeable (or even dominating in the large- $N_{c}$ limit) contribution from dimension 6 gluon condensate $\varepsilon^{a b c}\left\langle G_{a} G_{b} G_{c}\right\rangle$ which is not present in the phenomenological expansion (39). The latter appears after taking into account non-zero bare quark masses in the quark loops [68], i.e. from explicit breaking of chiral symmetry. Our model thus predicts that at low momenta the parton model logarithm in (39) acquires a contribution related with spontaneous chiral symmetry breaking (that might be related with formation of effective constituent quark mass) which is absent in the standard OPE constructed starting from the perturbation theory.

\section{Predictions from correlators at zero mo- mentum}

Now we will demonstrate how the same prediction $|\beta| \approx 0.3$ can follow from a simple analysis of vector correlator at zero momentum. Our key proposal is to treat the linear spectrum of SW model (14) as dual (in the sense of quark-hadron duality) to perturbation theory corrected by gluonic power terms in the OPE (39). These terms are known to appear from breaking of conformal symmetry. In other words, the spectrum (14) does not yet correspond to real resonances but appears as a result of "meromorphization" 
of perturbative background corrected at low energies by conformal symmetry breaking power terms. The hadron resonances are associated with deviations from a background. In our model, this means then that they are associated with non-zero intercept parameter $\beta$ in 35 .

Simultaneously $\beta \neq 0$ signifies the breaking of chiral symmetry - the appearance of contributions from quark condensate in the expansion (38) and of mass splitting between parity partners.

The given philosophy can be converted into a predictive calculational scheme. Consider the vector correlator (34) at zero momentum,

$$
\Pi(0)=-\frac{2 R}{g_{5}^{2}}|c|\left\{-\frac{1}{2}+\beta[\psi(1+\beta)+2 \gamma-1]\right\} .
$$

It is important to emphasize the role of our regularization: $\Pi(0)$ is a finite quantity with all constants fixed. In the standard regularization, the subtraction of infinite constant makes $\Pi(0)$ ambiguous.

The successful old hypothesis of Partial Conservation of Axial Current predicts in the chiral limit that the value of axial-vector correlator is

$$
\Pi_{A}(0)=f_{\pi}^{2}
$$

where $f_{\pi}$ is the weak pion decay constant emerging from the pion pole. In essence, one may regard (44) as an alternative definition of $f_{\pi}$ - if the spontaneous chiral symmetry breaking is assumed, the r.h.s. of (44) must give the residue of the corresponding Goldstone boson pole. The critical observation is that the axial-vector resonances should not contribute to $\Pi_{A}(0)$ because of a large mass gap in the axial channel $-f_{\pi}^{2}$ absorbs effectively all contributions to $\Pi_{A}(0)$. According to our philosophy, this means that terms with $\beta$ do not contribute. We get thus from (43) the equation for the axial intercept parameter $\beta_{a}$,

$$
\beta_{a}\left[\psi\left(1+\beta_{a}\right)+2 \gamma-1\right]=0 .
$$

This equation has two numerical solutions: $\beta_{a}=0$ (no $\operatorname{CSB}$ as $\langle\bar{q} q\rangle=0$, see the last term in $(38)$ ) and $\beta_{a} \approx 0.31$ which is almost exactly the value extracted above from the large $Q^{2}$ limit.

\footnotetext{
${ }^{4}$ In other models, the spectrum corresponding to perturbative background can be different. For instance, the spectrum of $S$-wave spin-1 mesons in light front holographic QCD behaves as $m_{n}^{2} \sim n+1 / 2$ [1], i.e. one should look for deviations from $\beta=-1 / 2$. It is curious to observe that this spectrum minimizes the power contributions to perturbative logarithm in the 2-point vector correlator within the class of linear spectra [46. The value of $\beta=-1 / 2$, however, is incompatible with positivity of gluon condensate (41) in our approach.
} 
When the equation (45) holds, we can obtain from (43), (44) and (40) the following relation for the slope of linear spectrum (35),

$$
4|c|=\frac{48 \pi^{2}}{N_{c}} f_{\pi}^{2}
$$

This relation for linear spectrum was derived in QCD sum rules in the large$N_{c}$ limit under various assumptions ${ }^{5}$ (see, e.g., Refs. [47]). The relation (46) meets well the phenomenology - in the real world with $N_{c}=3$, the empirical value (42) is reproduced for $f_{\pi}=87 \mathrm{MeV}$, which is the value of $f_{\pi}$ in the chiral limit according to the chiral perturbation theory [71]. We get the relation (46) in the opposite to the OPE based sum rules limit $Q^{2} \rightarrow 0$. In principle, we could act in the reverse direction - require (46) and obtain Eq. (45) as a consequence.

In the vector channel, the value of $\Pi_{V}(0)$ is also non-zero but the physical reason must be completely different - a conversion of $\omega$ and neutral $\rho$ mesons into massless photons and back is possible (the effect underlying the famous hypothesis of Vector Meson Dominance) leading to a kind of effective "photon" contribution. We do not know this contribution apriori, however, we can use the relation 72] (a form of the "Das-Mathur-Okubo sum rule" 73])

$$
-4 L_{10}=\left.\frac{d}{d Q^{2}}\left(\Pi_{V}-\Pi_{A}\right)\right|_{Q^{2}=0},
$$

where $L_{10}$ is one of constants of $S U_{f}(3)$ chiral Lagrangian 71,74 . From (34) we get

$$
\left.\frac{d}{d Q^{2}} \Pi\right|_{Q^{2}=0}=-\frac{R}{2 g_{5}^{2}}[\psi(1+\beta)+2 \gamma-1+\beta \psi(1,1+\beta)] .
$$

Using Eq. (45) and normalization (40) we finally obtain an equation for the vector intercept parameter $\beta_{v}$,

$$
\psi\left(1+\beta_{v}\right)+2 \gamma-1+\beta_{v} \psi\left(1,1+\beta_{v}\right)-\beta_{a} \psi\left(1,1+\beta_{a}\right)=\frac{96 \pi^{2} L_{10}}{N_{c}} .
$$

The typical values of $L_{10}$ extracted in the phenomenology lie near $L_{10} \approx$ $-5.5 \cdot 10^{-3} 74$. One should keep in mind, however, that the chiral constants

\footnotetext{
${ }^{5}$ The relation $(46)$ can be simply obtained 46 by combining the slope $2 m_{\rho}^{2}$ of spectra of Veneziano-like dual amplitudes with the relation $m_{\rho}^{2}=\left(24 \pi^{2} / N_{c}\right) f_{\pi}^{2}$ which often holds in models respecting the Vector Meson Dominance 69] including the classical QCD sum rules 68. It also emerges in attempts to incorporate the spontaneous CSB into the hadron string framework 70$]$.
} 
are scale dependent and the aforementioned values refer to the scale of $\rho$ meson mass, $L_{10}\left(m_{\rho}\right)$. But we should substitute to Eq. (49) the value at zero momentum. Fortunately the value of $L_{10}(0)$ can be determined in a scale-independent manner from hadronic $\tau$-decays. The extracted value is $L_{10}(0)=\left(-6.36 \pm\left. 0.09\right|_{\text {expt }} \pm\left. 0.16\right|_{\text {theor }}\right) \cdot 10^{-3}[75]$. With this value ${ }^{6}$ and $\beta_{a}=0.31$ the numerical solution of Eq. 49) yields $\beta_{v} \approx-0.26$ which is close to $\beta_{v} \approx-0.3$ estimated above (the exact agreement $\beta_{v}=-\beta_{a}$ is achieved at $\left.L_{10}=-7.5 \cdot 10^{-3}\right)$.

An alternative strategy for making fits can consist in writing equation for $N_{c}=3$ from 47 ) and (48),

$$
\psi\left(1+\beta_{v}\right)+\beta_{v} \psi\left(1,1+\beta_{v}\right)-\psi\left(1+\beta_{a}\right)-\beta_{a} \psi\left(1,1+\beta_{a}\right)=32 \pi^{2} L_{10},
$$

and imposing $\beta_{a}=-\beta_{v}$ to have a universal gluon condensate in the OPE (38). This would give $\beta_{a}=-\beta_{v} \approx 0.27$. After that we could reproduce a reasonable value of gluon condensate and observe a very small contribution of terms with $\beta_{a}$ to the axial correlator at zero momentum (we omit the general factor in what follows),

$$
\Pi_{A}(0) \sim-\frac{1}{2}+\beta_{a}\left[\psi\left(1+\beta_{a}\right)+2 \gamma-1\right] \approx-\frac{1}{2}-0.01
$$

while the corresponding contribution to the vector correlator would be relatively large,

$$
\Pi_{V}(0) \sim-\frac{1}{2}+\beta_{v}\left[\psi\left(1+\beta_{v}\right)+2 \gamma-1\right] \approx-\frac{1}{2}+0.27 .
$$

The given simple calculations demonstrate phenomenologically how the constant contributions to correlators which are interpreted as part of "contact" terms and neglected in high $Q^{2}$ expansions play a decisive role at low $Q^{2}$.

\section{Discussions}

There is a widespread opinion (see, e.g., discussions in Ref. [31]) that the phenomenology of bottom-up holographic models has much in common with

\footnotetext{
${ }^{6}$ An order by magnitude estimation of $S U_{f}(3)$ chiral constants is $L_{i} \sim \frac{f_{\pi}^{2}}{\Lambda_{\mathrm{CSB}}^{2}} 74$, where $\Lambda_{\mathrm{CSB}}$ is the scale of spontaneous chiral symmetry breaking. This scale is usually estimated from variation of contributions of chiral loops, the result is $\Lambda_{\mathrm{CSB}} \simeq 4 \pi f_{\pi}[76$ and leads to $L_{i} \sim 1 /(4 \pi)^{2} \approx 6.3 \cdot 10^{-3}$. Note that the slope of radial trajectories 46 at $N_{c}=3$ is just $\Lambda_{\text {CSB }}^{2}$. The given coincidence certainly should have deep physical roots.
} 
QCD sum rules in the large- $N_{c}$ limit (sometimes called planar QCD sum rules) which were a fruitful phenomenological tool in the past 36 47]. Within this method, one takes the pole representation of two-point correlators (3) in the Euclidean space,

$$
\Pi\left(Q^{2}\right) \sim Q^{2} \sum_{n=0}^{\infty} \frac{F_{n}^{2}}{Q^{2}+m_{n}^{2}},
$$

assumes some ansatz for the spectrum, sums over all states, matches the result with the corresponding OPE and low-energy constraints and finally derives phenomenological predictions. In the case of simple linear spectrum,

$$
m_{n}^{2}=\mu^{2}(n+b)
$$

with constant residues $F_{n}^{2}$ one obtains the usual representation via digamma function,

$$
\Pi\left(Q^{2}\right) \sim-\mathcal{Q}^{2} \psi\left(\mathcal{Q}^{2}+b\right)+\text { const }
$$

where $\mathcal{Q}^{2}=Q^{2} / \mu^{2}$.

We wish to emphasize an important technical distinction between this approach and our holographic one. In the vector case, we obtain a structure of the kind

$$
\Pi\left(\mathcal{Q}^{2}\right) \sim-\left(\mathcal{Q}^{2}+\beta\right) \psi\left(\mathcal{Q}^{2}+1+\beta\right)+\text { const. }
$$

Now changing intercept we change also the coefficient in front of $\psi$-function. As a result, the expansion in large $\mathcal{Q}^{2}$ of (56) becomes different from the expansion of (55) and consequently leads to a different set of sum rules when one matches to the OPE. The reason of arising distinction lies in the fact that the holographic models represent a dynamical approach where $\mathcal{Q}^{2}=-q^{2} / \mu^{2}$ appears in holographic e.o.m. like Eq. (21): Any shift of constant intercept $b \rightarrow b+\Delta b$ can be interpreted as a shift $Q^{2} \rightarrow Q^{2}+\mu^{2} \Delta b$ in the corresponding e.o.m., i.e. as a redefinition of what we call $]^{7}$ " $Q^{2}$ ". Physically this means, of course, a fine tuning of mass gap. It is interesting to note that this shift (i.e. $\beta \neq 0$ in (56) ) leads to the appearance of contribution $\beta \ln \mathcal{Q}^{2}$ in the large $\mathcal{Q}^{2}$ expansion (as in expansion (38)). As we remarked in the end of Sect. 2, this contribution should be related with the CSB in the form of acquired constituent quark mass. It is interesting to note that similar contributions were interpreted as contributions stemming from the effective non-local dimension-two gluon condensate in Refs. [77/79].

In our opinion, the $z$-dependent effective mass (18) should be viewed as primary while the dilaton background as secondary, i.e. the standard

\footnotetext{
${ }^{7}$ Actually this remark is valid for any extension of Klein-Gordon equation preserving the $4 \mathrm{D}$ relativistic invariance.
} 
SW holographic model follows after appropriate redefinition of fields. If this viewpoint is correct, a question appears concerning the physical origin of $z$ dependence from a more fundamental theory. Its origin might be inevitable when passing to non-conformal theories in the gauge/gravity correspondence. We remind the reader that a $4 \mathrm{D}$ physical state in a dual $10 \mathrm{D}$ theory emerges in the form

$$
\Phi=e^{i q x} \psi(z, \Omega)
$$

where $\Omega$ are coordinates on the $5 \mathrm{D}$ transverse space [4] which usually represents sphere $S_{5}$. In conformal case of pure $A d S_{5}$ space, a further factorization takes place,

$$
\psi(z, \Omega)=C v(z) g(\Omega),
$$

where $g(\Omega)$ is a normalized harmonic in the angular directions which can be safely integrated out. In non-conformal case, the factorization 58 is valid only at small $z$ because the infrared dynamics at large $z$ will in general induce mixing between different harmonics [4]. Nevertheless in AdS/QCD models, the factorization (58) is tacitly assumed for all $z$. The effective $z$-dependence of mass term might originate from this mixing after integration over $\Omega$. A holographic derivation of the form of this contribution remains of course an open problem.

Finally we would remark that the considered generalized SW model could find interesting applications in calculating various transition matrix elements (hadron form factors) and the gluon parton densities (parton distribution functions, transverse momentum distributions, and generalized parton distributions). These quantities are defined as certain overlap integrals of normalizable modes with boundary currents which propagate in AdS space [1]. It is known that calculations of these important physical quantities are usually rather successful in soft-wall AdS/QCD (see, e.g., a very recent work [80]). But we would expect a better quantitative agreement with the experimental data in case of considered generalized SW model due to to its flexibility in fine tuning the actual spectrum of mesons via the intercept parameter. Also our approach can be considered within the soft-wall Light-Front Holographic QCD [1 and help in solving the following problem: The poles of vector bulk-to-boundary propagator in time-like domain $\left(q_{n}^{2} \sim n+1\right)$ do not coincide with vector meson masses extracted from the bound-state equation $\left(m_{n}^{2} \sim n+1 / 2\right)$ and one needs to shift the pole positions manually to their physical location to obtain a meaningful comparison with measurements [1]. 


\section{Conclusions}

The main result of our work consists in an explicit demonstration of the fact that the AdS/QCD predictions for radial Regge spectrum of spin-1 mesons following from expansion of correlators at high momentum can be reproduced from expansion at low momentum. We provided a set of physical assumptions for which the whole scheme works successfully. Our analysis places on a new quantitative level the general idea that bottom-up holographic models interpolate between high and low energy sectors of QCD.

It is important to emphasize that according to the ideas of gauge/gravity duality, the semiclassical holographic dual models should describe the lowenergy domain where QCD is strongly coupled. This entails a much better conceptual justification for low-energy holographic predictions in comparison with the usual high-energy ones. The fact that such low-energy predictions can describe the whole radial spectrum looks encouraging.

Our approach can be extended to vector mesons interpolated by higher twist operators. This includes consideration of non-zero constant $a$ in the ansatz (18). In the case of arbitrary $a$, the structure of 2-point correlators becomes different and deserves a separate study. An extension to the scalar and tensor cases is straightforward. The development of corresponding phenomenology is in progress.

\section{Acknowledgments}

The work of S.S. Afonin is supported by the Russian Science Foundation, grant 21-12-00020 (the results of Section 2 - the high energy holographic constraints). The work of T.D. Solomko (the results of Section 3 - the low energy holographic predictions) was funded by RFBR grant for aspirants 19-32-90053.

\section{References}

[1] S. J. Brodsky, G. F. de Teramond, H. G. Dosch and J. Erlich, Phys. Rept. 584, 1 (2015).

[2] J. M. Maldacena, Adv. Theor. Math. Phys. 2, 231 (1998); Int. J. Theor. Phys. 38, 1113 (1999).

[3] E. Witten, Adv. Theor. Math. Phys. 2, 253 (1998); S. S. Gubser, I. R. Klebanov and A. M. Polyakov, Phys. Lett. B 428, 105 (1998). 
[4] J. Polchinski and M. J. Strassler, Phys. Rev. Lett. 88, 031601 (2002).

[5] J. Erlich, E. Katz, D. T. Son and M. A. Stephanov, Phys. Rev. Lett. 95, $261602(2005)$.

[6] L. Da Rold and A. Pomarol, Nucl. Phys. B 721, 79 (2005).

[7] A. Karch, E. Katz, D. T. Son and M. A. Stephanov, Phys. Rev. D 74, 015005 (2006).

[8] J. Erlich, G. D. Kribs and I. Low, Phys. Rev. D 73, 096001 (2006).

[9] H. Boschi-Filho, N. R. F. Braga and H. L. Carrion, Phys. Rev. D 73, 047901 (2006).

[10] J. Hirn, N. Rius and V. Sanz, Phys. Rev. D 73, 085005 (2006); K. Ghoroku, N. Maru, M. Tachibana and M. Yahiro, Phys. Lett. B 633, 602 (2006).

[11] C. Csáki and M. Reece, JHEP 0705, 062 (2007); J. P. Shock, F. Wu, Y.-L. Wu and Z.-F. Xie, JHEP 0703, 064 (2007).

[12] B. Batell and T. Gherghetta, Phys. Rev. D 78, 026002 (2008); T. Gherghetta, J. I. Kapusta and T. M. Kelley, Phys. Rev. D 79, 076003 (2009).

[13] A. Krikun, Phys. Rev. D 77, 126014 (2008);

[14] W. de Paula, T. Frederico, H. Forkel and M. Beyer, Phys. Rev. D 79, 075019 (2009).

[15] A. Vega and I. Schmidt, Phys. Rev. D 79, 055003 (2009).

[16] S. S. Afonin, Phys. Lett. B 675, 54 (2009); Phys. Lett. B 678, 477 (2009); Phys. Lett. B 809, 135780 (2020); Eur. Phys. J. C 71, 1830 (2011); Teor. Mat. Fiz. 184, 418 (2015); Mod. Phys. Lett. A 32, 1750155 (2017); Eur. Phys. J. C 80, no.8, 723 (2020); Acta Phys. Polon. Supp. 14, 109 (2021).

[17] G. F. de Teramond and S. J. Brodsky, Phys. Rev. Lett. 102, 081601 (2009); A. Cherman, T. D. Cohen and E. S. Werbos, Phys. Rev. C 79, 045203 (2009); D. Becciolini, M. Redi and A. Wulzer, JHEP 1001074 (2010). 
[18] S. Afonin, A. Andrianov and D. Espriu, Phys. Lett. B 745, 52 (2015); S. Afonin and I. Pusenkov, Phys. Lett. B 726, 283 (2013); S. Afonin and A. Katanaeva, Eur. Phys. J. C 74, 3124 (2014); Phys. Rev. D 98, 114027 (2018); arXiv:2009.05375 [hep-ph]; S. S. Afonin, A. D. Katanaeva, E. V. Prokhvatilov and M. I. Vyazovsky, Acta Phys. Polon. Supp. 13, $33(2020)$.

[19] T. Gutsche, V. E. Lyubovitskij, I. Schmidt and A. Vega, Phys. Rev. D 87, 056001 (2013); T. Gutsche, V. E. Lyubovitskij and I. Schmidt, Nucl. Phys. B 952, 114934 (2020).

[20] O. Andreev, Phys. Rev. D 73, 107901 (2006).

[21] H. Forkel, Phys. Rev. D 78, 025001 (2008).

[22] P. Colangelo, F. De Fazio, F. Giannuzzi, F. Jugeau and S. Nicotri, Phys. Rev. D 78, 055009 (2008); F. Jugeau, S. Narison and H. Ratsimbarison, Phys. Lett. B 722, 111 (2013).

[23] S. S. Afonin, Int. J. Mod. Phys. A 26, 3615 (2011).

[24] S. S. Afonin, Int. J. Mod. Phys. A 27, 1250171 (2012).

[25] S. S. Afonin, Phys. Lett. B 719, 399 (2013).

[26] H. Forkel, M. Beyer and T. Frederico, JHEP 0707, 077 (2007).

[27] N. Evans and A. Tedder, Phys. Lett. B 642, 546 (2006).

[28] S. S. Afonin, Phys. Rev. C 83, 048202 (2011); Eur. Phys. J. C 80, 723 (2020).

[29] N. R. F. Braga, M. A. Martin Contreras and S. Diles, Phys. Lett. B 763, 203 (2016).

[30] M. A. Martin Contreras and A. Vega, Phys. Rev. D 102, no.4, 046007 (2020).

[31] S. S. Afonin, Int. J. Mod. Phys. A 25, 5683 (2010); Adv. High Energy Phys. 2017, 8358473 (2017).

[32] A. Karch, E. Katz, D. T. Son and M. A. Stephanov, JHEP 1104, 066 (2011).

[33] F. Zuo, Phys. Rev. D 82, 086011 (2010); T. Gutsche, V. E. Lyubovitskij, I. Schmidt and A. Vega, Phys. Rev. D 85, 076003 (2012). 
[34] G. 't Hooft, Nucl. Phys. B 72, 461 (1974).

[35] E. Witten, Nucl. Phys. B 160, 57 (1979).

[36] M. Shifman, hep-ph/0009131; hep-ph/0507246.

[37] S. Beane, Phys. Rev. D 64, 116010 (2001).

[38] Yu. A. Simonov, Phys. Atom. Nucl. 65, 135 (2002).

[39] V. A. Andrianov and S. S. Afonin, Phys. Atom. Nucl. 65, 1862 (2002); S. S. Afonin, PMC Phys. A 3, 1 (2009).

[40] M. Golterman and S. Peris, JHEP 0101, 028 (2001); Phys. Rev. D 67, 096001 (2003); O. Cata, M. Golterman and S. Peris, JHEP 0508, 076 (2005).

[41] E. R. Arriola and W. Broniowski, Eur. Phys. 31, 739 (2007).

[42] J. J. Sanz-Cillero, Nucl. Phys. B 732, 136 (2006); J. Mondejar and A. Pineda, JHEP 0710, 061 (2007).

[43] A. A. Andrianov and D. Espriu, Phys. Lett. B 671, 275 (2009).

[44] S. S. Afonin and T. D. Solomko, Eur. Phys. J. C 76, 678 (2016); Int. J. Mod. Phys. A 33, 1850069 (2018); Int. J. Mod. Phys. A 33, 1850115 (2018); Eur. Phys. J. Plus 134, 10 (2019); Theor. Math. Phys. 200, 1075 (2019).

[45] S. S. Afonin, A. A. Andrianov, V. A. Andrianov and D. Espriu, JHEP 0404, 039 (2004).

[46] S. S. Afonin, Nucl. Phys. B 779, 13 (2007).

[47] S. S. Afonin, Phys. Lett. B 576, 122 (2003); S. S. Afonin and D. Espriu, JHEP 0609, 047 (2006).

[48] P.A. Zyla et al. (Particle Data Group), Prog. Theor. Exp. Phys. 2020, 083C01 (2020).

[49] R. Sundrum, Phys. Rev. D 86, 085025 (2012).

[50] J. K. Chen, Eur. Phys. J. C 78, no.8, 648 (2018); A. M. Badalian and B. L. G. Bakker, Phys. Rev. D 100, no.3, 034010 (2019). 
[51] A. V. Anisovich, V. V. Anisovich and A. V. Sarantsev, Phys. Rev. D 62, 051502(R) (2000); D. V. Bugg, Phys. Rept. 397, 257 (2004); E. Klempt and A. Zaitsev, Phys. Rept. 454, 1 (2007).

[52] D. M. Li, B. Ma, Y. X. Li, Q. K. Yao and H. Yu, Eur. Phys. J. C 37, 323 (2004).

[53] S. S. Afonin, Phys. Lett. B 639, 258 (2006); Eur. Phys. J. A 29, 327 (2006); Phys. Rev. C 76, 015202 (2007); Int. J. Mod. Phys. A 22, 4537 (2007); Int. J. Mod. Phys. A 23, 4205 (2008); Mod. Phys. Lett. A 22, 1359 (2007); Mod. Phys. Lett. A 23, 4205 (2008); Mod. Phys. Lett. A 23, 3159 (2008).

[54] M. Shifman and A. Vainshtein, Phys. Rev. D 77, 034002 (2008).

[55] P. Masjuan, E. Ruiz Arriola and W. Broniowski, Phys. Rev. D 85, 094006 (2012).

[56] S. S. Afonin and I. V. Pusenkov, Phys. Rev. D 90, 094020 (2014); Mod. Phys. Lett. A 29, 1450193 (2014); S. S. Afonin, arXiv:2009.05378 [hepph].

[57] D. LaCourse and M. G. Olsson, Phys. Rev. D 39, 2751 (1989); T. J. Allen, C. Goebel, M. G. Olsson and S. Veseli, Phys. Rev. D 64, 094011 (2001).

[58] A. Yu. Dubin, A. B. Kaidalov and Yu. A. Simonov, Phys. Lett. B 323, 41 (1994); Yu. S. Kalashnikova, A. V. Nefediev and Yu. A. Simonov, Phys. Rev. D 64, 014037 (2001); A. M. Badalian, B. L. G. Bakker and Y. A. Simonov, Phys. Rev. D 66, 034026 (2002).

[59] G. V. Efimov and S. N. Nedelko, Phys. Rev. D 51, 176 (1995).

[60] R. Ricken, M. Koll, D. Merten, B. C. Metsch and H. R. Petry, Eur. Phys. J. A 9, 221 (2000).

[61] M. Baker and R. Steinke, Phys. Rev. D 65, 094042 (2002).

[62] F. Buisseret, Phys. Rev. C 76, 025206 (2007).

[63] D. Ebert, R. N. Faustov and V. O. Galkin, Phys. Rev. D 79, 114029 (2009).

[64] J. Sonnenschein and D. Weissman, JHEP 1408, 013 (2014). 
[65] S. S. Afonin, Adv. High Energy Phys. 2019, 1701939 (2019).

[66] D. Jia, C. Q. Pang and A. Hosaka, Int. J. Mod. Phys. A 32, 1750153 (2017).

[67] K. Skenderis, Class. Quant. Grav. 19, 5849 (2002).

[68] M. A. Shifman, A. I. Vainstein and V. I. Zakharov, Nucl. Phys. B 147, 385, 448 (1979).

[69] E. R. Arriola and W. Broniowski, Phys. Rev. D 67, 074021 (2003).

[70] A. Andrianov, D. Espriu and A. Prats, AIP Conf. Proc. 756, 302 (2005) arXiv:hep-ph/0412380 [hep-ph]].

[71] J. Gasser and H. Leutwyler, Nucl. Phys. B 250, 465 (1985).

[72] G. Ecker, J. Gasser, A. Pich and E. de Rafael, Nucl. Phys. B 321, 311 (1989).

[73] T. Das, V.S. Mathur and S. Okubo, Phys. Rev. Lett. 19, 859 (1967).

[74] A. Pich, arXiv:1804.05664 [hep-ph].

[75] M. Davier, A. Hocker, L. Girlanda, and J. Stern, Phys. Rev. D 58, 096014 (1998).

[76] A. Manohar and H. Georgi Nucl. Phys. B 234, 189 (1984); S. Weinberg, Physica A 96, 327 (1979).

[77] F. V. Gubarev and V. I. Zakharov, Phys. Lett. B 501, 28 (2001); K. G. Chetirkin, S. Narison, and V. I. Zakharov, Nucl. Phys. B 550, 353 (1999); S. Narison and V. I. Zakharov, Phys. Lett. B 522, 266 (2001).

[78] E. R. Arriola and W. Broniowski, Phys. Rev. D 73, 097502 (2006); 81, 054009 (2010).

[79] V. I. Shevchenko and Yu. A. Simonov, Phys. Rev. D 70, 074012 (2004).

[80] V. E. Lyubovitskij and I. Schmidt, arXiv:2012.01334 [hep-ph]. 